\title{
Entwicklung und Situation der Medienpädagogik im deutschsprachigen Raum
}

\author{
von Ingrid Geretscblaeger
}

Der vorliegende Aufsatz basiert auf den theoretischen Einleitungskapiteln der Dissertation der Verfasserin. Die Arbeit „Analyse der medienpädagogischen Literatur 1970 bis 1976" wurde im Frühjahr 1977 am Salzburger Institut für Publizistik und Kommunikationstheorie abgeschlossen und beschäftigt sich in ihrem Hauptteil mit der Analyse von 249 Publikationen aus der Bundesrepublik Deutschland, der Deutschen Demokratischen Republik, Osterreich und der Schweiz nach Kriterien, die Aufschluß geben über die Art der Vermittlung und die Inhalte aus dem Bereich der Massenkommunikation im Feld der Pädagogik.

Medienpädagogik, wie immer man sie auffassen mag, ist eine Spezialform der Pädagogik. Vom Verständnis der Pädagogik hängt es ab, wie weit oder wie eng der Vermittlungsbereich gefaßt wird; vom Begriff des Mediums, welche Bestimmung diese Pädagogik erfährt.

Trotz der Zugehörigkeit zur Pädagogik konstituiert also die Begriffsbestimmung Medium den Inhalt der Medienpädagogik. Beschränkt man sich auf ein Medium (hier ist vom publizistikwissenschaftlichen Begriffsinhalt der Massenmedien die Rede), so spricht man von Presse-, Film-, Fernseh- oder Hörfunkerziehung bzw. -pädagogik.

Die Pädagogik kann sich nun je nach ihrem Verständis mit den Medien in positiver oder ablehnender Haltung beschäftigen. Als positive Einstellung wäre die Erziehung z.u einem Medium (zu den Medien) und auch der Einsatz von Medien für unterrichtliche $Z_{\text {wecke }}$ zu sehen, wenngleich dies nicht in die engere Definition von Medienpädagogik fällt, jedoch geschichtlich relevant ist, als ablehnende Haltung müßte man jene bezeichnen, die das Fernhalten des schlechten Einflusses des Mediums (der Medien) zum Ziel hat.

Diese verschiedenen Ausprägungen wechseln sich ab, können aber auch nebeneinander liegen, je nach der Einstellung des Sozialisators (Erziehers, Lehrers) gegenüber den Medien.

\section{Geschichte}

Die Anfänge der Medienpädagogik können theoretisch dort liegen, wo Massenmedien bereits vorhanden sind. Zeitungen und Zeitschriften müßten also die ersten gewesen sein, denen man sich zuwandte. Tatsächlich aber verwendete man zwar Zeitungen für den Wissenszuwachs, doch sie zu hinterfragen war nicht im Sinn der Erzieher.

Beim Film lag das etwas anders. Der war etwas Geheimnisvolles, von dem man nicht recht wußte, was man davon halten sollte. Sind Kinovorstellungen etwas Schlechtes,

Dr. phil. Ingrid Geretschlaeger hat nach mehrjähriger Berufstätigkeit als Grundschullehrerin ihre praktischen Erfahrungen in der Medienerziehung in einem Studium der Publizistikwissenschaft wissenschaftlich ausgewertet. Sie arbeitet derzeit als freie wiss. Mitarbeiterin in Salzburg. 
oder lernt man dabei etwas - die Geister schieden sich: die einen lehnten Film ab, die anderen begannen, ihn für sich zu nutzen.

Der Nationalsozialismus gab dem Film die Stellung, die ihm in diesem Sinn gebührte - nämlich dort, „... wo das bewegte Bild eindringlicher als alles andere zum Kinde spricht" 1 .

25 Jahre später sagte man über den Film: „Wir wissen heute, daß diese suggestive Kraft eine der wesentlichsten Ursachen dafür ist, daß viele der bewegten Bilder der Leinwand, die in der Lage sind, uns zu bannen, wegen ihrer starken emotionalen Wirkungen direkt in das menschliche Unterbewußtsein gelangen. “2

Mit der Hilfe von Filmgesprächen sollten diese Wirkungen neutralisiert werden bewußt gemacht werden. Man strebte eine Desillusionierung der Jugendlichen in bezug auf den Film an, hatte aber gleichzeitig Angst vor der Gefahr, damit die Erlebnisfähigkeit zu zerstören ${ }^{3}$.

Bald auch mußte man sich der Fernseherziehung zuwenden, und man tat dies mit den Kriterien der Filmerziehung, obwohl gerade das Fernsehen neben dem Hörfunk ein Medium war, das den Weg in die Schule über Schulfunk und Schulfernsehen fand. In dieser Form aber lernen Jugendliche, Informationen vom Bildschirm bzw. über den Äther kritikios zu übernehmen. Wirkt das nicht den pädagogischen Bemühungen zur Kritikfähigkeit entgegen? Kerstiens lehnte es schon 1959 ab, mit Hilfe von Filmen Jugendliche zu erziehen. Er forderte, daß alles Geschehen Gegenstand der Bewertung sein müsse

Massenmedien finden immer weiter Eingang in Erziehung und Bildung. Schließlich kommt man nicht mehr darum herum, alle Massenmedien in die Betrachtung einzubeziehen. Medienerziehung wird „... vor dem Hintergrund einer gesamtpädagogischen Konzeption und eines umfassenden pädagogischen Bemühens . . " ${ }^{5}$ gesehen.

In der Folge fordert man den Ubbergang zur allgemeinen Kommunikationserziehung, die alle Kommunikationsprpozesse (die ,intra-" und ,interpersonalen ${ }^{c}{ }^{\theta}$ miteinbezieht. Diese Kommunikationserziehung nähert sich aber den Bereichen der Gruppendynamik an. Massenmedien sollen jedoch in ihrer Funktion als Massenmedien betrachtet werden und nicht in ihrer dienenden Funktion als Auslöser von Gruppenkommunikationsprozessen. Sie sind ein wesentlicher Bestandteil unserer Gesellschaft geworden und müssen in ihrer Funktion für die Gesamtheit der Rezipienten hinterfragt werden. Massenkommunikation muß gelehrt und gelernt werden.

Medienarbeit, Medienkritik - Schlagworte zu Beginn der 70er Jahre; Emanzipatorischer Mediengebrauch, Transparenz der Medien - das sind Zielvorstellungen, die in marxistischen wie religiös orientierten medienpädagogischen Gruppierungen auftauchen.

\section{Situation}

Erziehung und Bildung ist in der Bundesrepublik Deutschland Ländersache, in der Deutschen Demokratischen Republik und in Osterreich werden die Bestimmungen zentral verordnet, in der Schweiz ist sie Aufgabe der Kantone.

Ein weiterer Unterschied ergibt sich aus der Einstellung gegenüber der Massenkommunikation, was entweder eine den Massenmedien gegenüber grundsätzlich kritische Haltung bedingt oder eine sehr positive aus Gründen des politischen Lernens vom 
Standpunkt der sozialistischen Ideologie. Ein Ubergewicht der medien-politischen gegenüber der medienpädagogischen Einstellung läßt sich in der Deutschen Demokratischen Republik feststellen; ähnliche Tendenzen finden sich aber auch in den westlichen Ländern: Kompromißlose Kritik an Produkten abzulehnender bzw. zu bekämpfender Ideologien steht die positive Bewertung der eigenen Botschaft mit der positiven Absicht der Erziehung der Massen gegenüber.

\subsection{Bundesrepublik Deutschland}

In den Schulen der Bundesrepublik Deutschland hat sich die Medienpädagogik bzw. Medienerziehung nicht als eigenes Fach durchgesetzt. Die meisten Versuche sind aus schulorganisatorischen, aber auch aus didaktisch-methodischen Gründen gescheitert. Medienerziehung wird folglich als fächerübergreifendes Prinzip gehandhabt ${ }^{7}$.

Den Lehrplänen aller Bundesländer gemeinsam ist die Behandlung der Massenmedien im Deutschunterricht; in acht Ländern zusätzlich in Sozialkunde/Politische Bildung/ Gegenwartskunde/Gemeinschaftskunde; fünfmal in Bildende Kunst/Visuelle Kommunikation; einmal im Fach Evangelischer Religionsunterricht ${ }^{8}$.

Die Themen für die unterrichtliche Behandlung sind breit gestreut: „Film als $\mathrm{Me}$ dium“, „Fernsehen und Wirklichkeit“, "Sprache als Mittel der Information und Manipulation“, „Umgang mit Texten“, „Kommunikationsmodelle“, „Kommunikationsstrukturen“, „Umgang mit Massenmedien und die Auseinandersetzung mit den Masseneinflüssen“, „Medienerziehung“, „Massenmedien als Unterrichtsgegenstand“.

Medienerziehung bzw. Medienpädagogik als Teil einer umfassenden Kommunikationserziehung gibt es noch an den Fachschulen und Fachbochschulen für Sozialpädagogik in den Bundesländern Bayern, Berlin, Niedersachsen, Nordrhein-Westfalen, Rheinland-Pfalz und dem Saarland, aber mit jeweils sehr unterschiedlicher Schwerpunktsetzung9. An der Universität Osnabrück kann seit dem Wintersemester 1977/78 das Fach Medien als Magister-Studiengang voll studiert werden ${ }^{\mathbf{1 0}}$.

Für den Bereich der Erwachsenenbildung haben Breuer/Hüther festgestellt, „... daß man hier den Massenmedien als Unterrichtsgegenstand ziemlich hilflos gegenübersteht $^{\text {“11. }}$. Für diesen Bereich wurde vom FWU (Institut für Film und Bild in Wissenschaft und Unterricht) ein Lernsystem „Medienkunde“ als Medienpaket produziert. Derzeit ist ein Projekt „Kommunikationslehre“ im letzten Stadium der Ausarbeitung ${ }^{12}$.

Auf Bundes- und Landesebene werden im Bereich Medienpädagogik auch die Zentralen für Politische Bildung tätig. Die Bundeszentrale z. B. beteiligt sich an verschiedenen medienpädagogischen Projekten bzw. initiiert sie („Prüfung vor Ort“ -1976 ; "Jugendliche analysieren den Schüler-Express" - 1976/77; "Serienwerkstatt '73“ und deren Nachseminar 1977). Als Herausgeber der Wochenzeitung „Das Parlament “ mit der ständigen Seite "Teleforum“ leistet sie auch einen Beitrag zur Medienpädagogik in den Massenmedien selbst.

Als kleinere Institutionen seien das „Institut Jugend Film Fernsehen “ mit der Zeitschrift „medien + erziehung “ und das „Medienpädagogik Zentrum“ mit den „mpzmaterialien“ und den „mpz-Informationen“ genannt.

$\mathrm{Zu}$ den bedeutenden Trägern der Medienarbeit gehören zweifelsohne auch die beiden Kirchen. So leistet beispielsweise das "Gemeinschaftswerk der Evangelischen Publizistik“ mit den vielen Veranstaltungen zur Medienpädagogik, mit der Zeitschrift 
„medium“, die häufig medienpädagogische Themen aufgreift, und mit umfangreichen Materialien zu Seminaren einen sehr wesentlichen Beitrag.

Auf katholischer Seite kann als ein Beispiel die „Kirchliche Zentralstelle für Medien“ genannt werden, die mit ihrem Referat Kommunikationspädagogik eine Reihe von Schriften (z. B. "medienpraxis") herausgibt.

Massenmedien beschäftigen sich mit Massenmedien nicht gerade häufig Das Fernsehen als das umfassendste Medium findet dabei noch die größte Beachtung.

So sind es neben den Zeitschriften, die sich in wissenschaftlicher oder fachlicher Beziehung mit Kommunikationsbelangen beschäftigen, vor allem Programmzeitschriften, die sporadisch medienpädagogisch zu nennende Beiträge veröffentlichen. Für Magazine und große Illustrierte ist Medienpädagogik kein publikumswirksamer Stoff.

Die medien(kritische) Leiste der ARD ist bisher wenig ausgebaut und eine explizit medienpädagogische Sendung existiert gar nicht. „Mikado“ und "Glashaus - tv intern" schneiden medienkundliche Aspekte des Fernsehens an - pressekritische Sendungen sind geplant ${ }^{13}$.

Eine Programmgattung, die Informationen über Massenmedien liefert, gibt es bisher nur in weniger publikumswirksamen Programmen der Landesanstalten NDR, BR sowie SWF mit ihren dritten Programmen und beim WDR und SFB mit ihren Schulfernsehprogrammen, die auch vom BR übernommen werden. Besonders der WDR, der seit 1975 über eine Redaktion „Medienerziehung “ verfügt, hat das seit 1973/74 laufende Projekt „Schüler machen Filme“ (jetzt „Arbeitsgemeinschaft Schülerfernsehen ${ }^{\text {) }) ~ s y s t e m a t i s i e r t ~ u n d ~ e r g a ̈ n z t . ~}$

Das ZDF strahlt seit 1974 die medienkundliche Reihe „betrifft: fernsehen“ mit dem Ziel aus, einen urteilsfähigen Zuschauer heranzubilden. In der oftmals kritisierten Reihe „... soll das Fernsehen sich selbst mit seinen Vorzügen und Schwächen, Zwängen und Möglichkeiten darstellen “14.

\subsection{Deutsche Demokratische Republik}

Um die Form der Medienerziehung in der DDR verstehen zu können, muß man sich vorerst darüber im klaren sein, was unter Massenkommunikation und Massenmedien verstanden wird, sowie welches Ziel der Erziehung vorangestellt ist.

Die ideologische Massenkommunikation im Sozialismus wird „... als ein mit Hilfe der modernen Informationsmittel bewußt geleiteter und systematisch gestalteter Prozeß der sozialistischen Bewußtseinsbildung in allen Klassen und Schichten, darunter der Jugend aufgefaßt. In ihrem Mittelpunkt steht die Verbreitung und Aneignung der sozialistischen Ideologie in effektiven massenwirksamen Formen . . ."15

Jede Form der Erziebung strebt also hin auf das allgemeine Ziel der sozialistischen Persönlichkeit, die erfaßt sein muß von der sozialistischen Ideologie, die geistiges und praktisches Handeln durchdringt. Dabei bestimmen die Erfordernisse des Klassenkampfes die Inhalte der politischen Erziehung ${ }^{\mathbf{1 6}}$.

Damit es zu der geforderten Wirkung kommt, müssen die Jugendlichen die Massenmedien in entsprechender Weise nutzen. Sie müssen sich die Inhalte in aktiver Weise aneignen. Dafür werden drei Komponenten als ausschlaggebend bezeichnet: 1. Fähigkeit zur richtigen klassenmäßigen Auswahl aus dem medialen Angebot (Selektionsfähigkeit), 2. Fähigkeit zur Aneignung der von den Massenmedien gelieferten Infor- 
mationen zum richtigen Verstehen und Verwerten derselben (Reproduktionsfähigkeit) und 3. Fähigkeit, die Botschaft der Massenmedien richtig $\mathrm{zu}$ verarbeiten und die entsprechenden Konsequenzen für das eigene Handeln und Verhalten daraus zu ziehen $^{17}$.

"In der sozialistischen Gesellschaft geht es nicht darum, Jugendliche vor den ,geheimen Miterziehern' zu schützen, sondern in erster Linie müssen Konzeptionen entwickelt und realisiert werden, die den jugendlichen Rezipienten zu einem für die sozialistische Persönlichkeitsformung und Bewußtseinsentwicklung effektiven Gebrauch der Massenkommunikation befähigen ${ }^{\alpha 18}$, sagt Bisky.

Neben der ideologischen Grundposition, die jeder Jugendliche erwerben soll, gehört dazu auch „... ein gewisses Maß an Wissen über Inhalt, Methode und Techniken ideologischer Diversion und Kriegsführung, um den Gegner in jeder Situation ,durchschauen' $^{\mathrm{zu}}$ lernen "19.

Bisky und Süße formulieren den Anspruch der Medienerziehung in der Deutschen Demokratischen Republik so: „Sie müßte ein bestimmtes Maß an Grundwissen über die Struktur und Funktion der Massenmedien in der sozialistischen Gesellschaft, über den Mißbrauch im Imperialismus, über das Entstehen und Verbreiten von Nachrichten, über die spezielle Funktion der Massenmedien im ideologischen Kampf ebenso umfassen wie spezielle Filmerziehung. ${ }^{\text {"20 }}$

Man erwartet vom Lebrer, den Einfluß der Massenmedien für die sozialistische Persönlichkeitsentwicklung zu lenken. Falsches Herangehen könnte hier ihren Einfluß wertlos machen ${ }^{21}$.

Großer Wert wird also auf die Didaktik der Medien gelegt. Seminare für Deutschlehrer an den Pädagogischen Hochschulen haben als Schwerpunkte: „Stellung von Film und Fernsehen in der heutigen Gesellschaft", „Anteil des künstlerischen Films und der Fernsehspiele bei der Entwicklung von Bewußtsein und Charakter der heranwachsenden sozialistischen Persönlichkeit“, „Pädagogisch-psychologische Grundlagen der Film- und Fernsehrezeption und methodische Grundüberlegungen zu schulischem und außerschulischem Einsatz von Film und Fernsehspielen ${ }^{\text {“22 }}$. Durch diese Seminare soll die ideologische Urteilsbildung des Lehrers gefördert werden.

Die Presse als „Instrument der ideologischen Arbeit der Partei bei der Herausbildung allseitig entwickelter Persönlichkeiten ${ }^{\alpha 23}$ ist der zweite, intensiv betriebene Bereich der medienerziehlichen Arbeit. Sie wird in den Schulen ab der vierten und fünften Klasse als Erziehung zum wichtigsten Informationsmittel systematisch durchgeführt ${ }^{24}$.

Es heißt parteilich zu sein für den Sozialismus und gegen den Kapitalismus. Die Medienerziehung muß daher beide Aspekte berücksichtigen: einerseits die richtige Nutzung im Rahmen des Sozialismus, andererseits die Kritik und Ablehnung gegenüber den westlichen Massenmedien. Beides aber kann nur durch die rechte - sozialistische - Gesinnung erreicht werden.

\subsection{Osterreich}

Die Situation der Medienpädagogik in Osterreich ist gekennzeichnet durch den sogenannten Medienerla $\beta$ des Bundesministeriums für Unterricht und Kunst aus dem Jahr 1973. Dieser Erlaß besagt, daß jeder Lehrer verpflichtet ist, auf die Medienerziehung „... als Unterrichtsprinzip in allen Unterrichtsgegenständen fachspezifisch 
Bedacht zu nehmen "25. Ziel ist es, zu einem „... bewußten und mitbestimmenden Konsumverhalten zu führen. Der Schüler soll durch die Medienerziehung befähigt werden, sich in einer Welt zurechtzufinden, über die er zum großen Teil durch Massenmedien informiert wird. ${ }^{* 26}$

Eingang findet die Medienerziehung so besonders in den Bereichen Sachunterricht und Deutsch der Volks- und Hauptschule, sowie im Fach Deutsch und Politische Bildung an höheren Schulen, obgleich sich auch Hinweise in den allgemeinen didaktischen Grundsätzen fast aller Lehrpläne finden.

Die gesamtösterreichischen Aktivitäten werden im „Zentralen Arbeitsausschuß für Medienerziehung in Schulen“ erfaßt, in den je zwei Vertreter der Landesarbeitskreise bei den Landesschulräten der einzelnen Bundesländer entsandt werden ${ }^{27}$.

Seit dem Schuljahr 1976/77 besteht die Möglichkeit der „Unverbindlichen Úbung“ "Medienkunde“ mit zwei Wochenstunden in den sechsten und siebten Klassen der Allgemeinbildenden höheren Schulen.

An den Pädagogischen Akademien des Bundes gab es seit 1968 als Unterrichtsfach die „Pädagogische Medienkunde“ mit einer Wochenstunde. Diese wird seit Herbst 1976 durch den zweistündigen Kurs „Unterrichtstechnologie“ ersetzt. Hierfür ist vorgesehen, neben der Didaktik und Methodik der technischen Unterrichtsmedien auf die "Integration der Medienerziehung in den Unterricht" sowie die "Analyse von Massenmedien nach pädagogischen, psychologischen, soziologischen und ästhetischen Aspekten ${ }^{\text {28 }}$ einzugehen.

Die spezielle medienpädagogische Ausbildung der Lehrer ist im allgemeinen sehr mangelhaft. Den Großteil medienpädagogisch Tätiger bilden Autodidakten oder Lehrer, die freiwillig an Kursen der Aktion "Der gute Film", der „Arbeitsgemeinschaft Jugend und Massenmedien“ oder des Instituts für Kommunikationswissenschaft teilgenommen haben. Das Bundesministerium für Unterricht und Kunst veranstaltet seit kurzem auch Wochenseminare für AHS-Lehrer.

Für die Lehrerfortbildung soll aber ansonsten an Pädagogischen Instituten gesorgt werden, wobei häufig mit der Aktion „Der gute Film“ zusammengearbeitet wird ${ }^{20}$.

Von privater Seite ist es besonders die Katholische Kirche mit dem Zentrum für Massenkommunikation und der Zeitschrift "Multimedia“, die sich intensiv der $\mathrm{Me}$ dienerziehung annimmt, sowohl in der Ausbildung der Priester als auch in der Lehrerbildung.

In der Jugendarbeit der Kirche wurde 1975/76 der Schwerpunkt auf Medienerziehung gelegt. Die Erziehungs- und Bildungsziele für die außerschulische kirchliche Kinderarbeit enthalten Medienerziehung als wichtigen Punkt in allen Altersgruppen ${ }^{30}$.

Der ORF startete im Herbst 1976 den Versuch einer medienkritischen Reihe. „Apropos TV" sollte zweimonatlich hinter die Kulissen des Fernsehens schauen: z. B. Wie wird eine TV-Serie in der Bundesrepublik Deutschland, wie in den USA produziert ${ }^{31}$ ?

Vereinzelte Beiträge zur Medienerziehung gibt es im österreichischen Schulfernsehen z. B. im Bereich Deutsch der achten Schulstufe "Was ist ein Film?"

Eine Sonderaktion des BMfUK im Herbst 1977 zum Nationalfeiertag am 26. Oktober stand im Zeichen "Massenmedien und Politische Bildung“. Presse, Hörfunk und Fernsehen wirkten mit - den Schülern wurde Gelegenheit gegeben, Eigenproduktionen 
vorzustellen oder auch nur Einblicke in die betreffenden Institutionen zu bekommen. Die Hauptaktivität der Schüler blieb jedoch - neben lobenswerten Ergebnissen bei der Gestaltung eigener Programme im Hörfunk - das Herstellen von Wand- oder Schülerzeitungen.

\subsection{Schweiz}

Medienerziehung in der Schweiz reicht von der rein technischen Seite des Einsatzes, von Medienerziehung im Fach Lebenskunde, über Film- und Fernseherziehung bis hin zur obligatorischen Medienerziehung mit fünf oder zehn Stunden pro Schuljahr bzw. zur Massenmedienkunde.

Der Schwerpunkt in der Thematik helvetischer Lehrpläne liegt bei Film und Fernsehen in den Fächern Deutsch und Lebenskunde. Zentrum der Beschäftigung mit Massenmedien ist die Oberstufe der Volksschule, doch findet man sie bereits in der Primarschule.

In einigen Kantonen wurden in letzter Zeit Entwürfe für eine Medienerziehung erarbeitet; so im Kanton Basel-Stadt, wo eine Projektstudie für ein Konzept für Medienerziehung in der Schule anhand von konkreten schulischen Projekten durchgeführt wurde ${ }^{32}$. Der erste praktische Schritt sollte die Lehrerbildung sein. Ein Ziel ist die Institutionalisierung der Medienerziehung in den Lehrplänen.

In St. Gallen gilt seit dem Frühjahr 1976 Medienerziehung verpflichtend für die Sekundarschulen, Appenzell wird sich bei der Revision der Lehrpläne denen des Kantons St. Gallen anschließen.

Das angestrebte Verhältnis zu den Massenkommunikationsmitteln wird im Lehrplan für Realschulen von Luzern und Nidwalden so dargestellt: „Die MKM haben immer eine dienende Funktion und übernehmen die Aufgabe der Meinungsbildung. Sie wollen also den modernen Menschen zu kritischer Einstellung befähigen und zur eigenen Meinungsbildung provozieren, indem sie ihn mit unterschiedlichen, of $t$ gegensätzlichen Meinungen konfrontieren. Notwendig und unerläßlich ist es, die Menschen der Gegenwart zu einem vernünftigen Gebrauch der MKM anzuleiten, zu erziehen und aufzufordern. “33

Die Lehrer werden zum Teil an Lebrerseminaren in Freifachkursen mit einer Wochenstunde auf ihre Arbeit vorbereitet und/oder in Lehrerfortbildungskursen bzw. in Form von Kaderschulungen. An der Kaderschulung der „Aus- und Weiterbildung von medienpädagogisch Tätigen" beteiligten sich neben Institutionen kirchlicher $\mathrm{Me}$ dienarbeit auch andere Institutionen wie die "Schweizerische Arbeitsgemeinschaft Jugend und Massenmedien“, die aber im Frühjahr 1977 aufgelöst wurde.

Die av-Zentrale des Pestalozzianums wurde damit beauftragt, einen Rahmenplan für die Medienerziehung im Kanton Zürich bzw. für die Medienerziehung in der gesamten Schweiz zu erstellen ${ }^{34}$. Sie ist auch in der Lehrerbildung tätig.

Doelker schreibt über die Lage der Medienpädagogik in der Schweiz, daß das, was in der Schule „... der Eigeninitiative von Lehrern zuzuschreiben ist, im allgemein pädagogischen und erzieherischen Bereich in der Schweiz fast immer dem Zusammenwirken öffentlicher und privater Kreise zugewiesen werden kann. Damit zeigt sich auch, daß sich die Schule in der Schweiz viel gesellschaftsbezogener entwickelt als dies manchmal angenommen wird. ${ }^{\text {(ss }}$ 
Auch die Massenmedien werden - wie die "Neue Zürcher Zeitung" mit ihrer intensiven Auseinandersetzung mit massenmedialen Themen - selbst medienpädagogisch wirksam, oder sie beteiligen sich an medienpädagogischen Aktivitäten, wobei sie vorwiegend mit Schulgruppen zusammenarbeiten.

Im Jugendfernsehen des DRS lief im Januar 1975 eine 13teilige filmkundliche Sendereihe unter dem Titel „Achtung, Aufnahme“ an. Ziel dieser Reihe soll es sein, „... die zu Hause vor dem Bildschirm sitzenden jungen Zuschauer im Alter von 12 bis 16 Jahren so zu motivieren, daß sie sich zusammentun, um sich selbst an die Realisierung eines kleinen Films zu wagen “"38.

Bereits seit 1974 gibt es eine Medien-„Aufklärungs“-Serie „Fernsehstraße 1-4". "Vom Medium Buch zum Medium Film“ heißt eine TV-Sendung für Jugendliche, die im Medienverbund in der Schule eingesetzt werden kann. Weitere Aktivitäten sind in einem neu geschaffenen TV-Magazin "Karussell“ und in medienkundlichen Bausteinen für das Jahr 1978 vorgesehen ${ }^{37}$.

Auch das Schweizerische Schulfernsehen strahlt medienkundliche Sendungen aus, so z. B. "Wie eine Radiosendung entsteht".

Selbstverständlich gibt es in allen hier beschriebenen Ländern zusätzliche medienpädagogische Aktivitäten, auf die hier nicht näher eingegangen werden kann. Außerdem laufen Projekte, die eine landesweite Ausbreitung anstreben und somit auf eine Vereinheitlichung der medienpädagogischen Tätigkeit abzielen. Kennzeichnend für sie ist, daß Medienpädagogik in keinem Fach angesiedelt wird, sondern immer interdisziplinär konzipiert ist. Zudem dominiert der medienübergreifende Aspekt. Projektunterricht wird gefordert, der in Form von einzelnen Steinen den Bereich der Massenkommunikation abdecken soll.

Aufbauend auf diesem allgemeinen Kern wird in der Bundesrepublik Deutschland ein Curriculum für die Sekundarstufe I zusammengestellt, in Osterreich ist es ein Projekt für die Multiplikatorenbildung mit direkter Übertragbarkeit in die Praxis, in der Schweiz soll der gesamte Schulbereich - inklusive Vorschule und Lehrerbildung - durch Curricula abgedeckt werden.

Die Deutsche Demokratische Republik muß ausgeklammert bleiben, da es dort soweit dies in Erfahrung gebracht werden konnte - kein vergleichbares Projekt gibt.

Anmerkungen:

1. Min. Erl. v. 26. Juni 1934, zit. nach: Zierold, Kurt: Bestimmungen über Film und Bild in Wissenschaft und Unterricht - mit Erläuterungen von Dr. Kurt Zierold (Schriftenreihe der Reichsstelle für Film und Bild in Wissenschaft und Unterricht). Kohlhammer, Stuttgart und Berlin, ${ }^{3} 1940$, S. 1-7, hier S. 1.

2. Mohrhof, Siegfried: Filmgespräche mit Jugendlichen. In: Mohrhof, Siegfried (Hrsg.): Filmgespräche mit Jugendlichen. Beiträge zur Methodik und Praxis der Jugendfilmarbeit. Institut für Film und Bild in Wissenschaft und Unterricht. München 1960, S. 5-13, hier S. 6. 
3. Vgl. dazu: Chresta, Hans: Filmerziehung in Schule und Jugendgruppe - Grundlagen, Methode, Arbeitsunterlagen (Schriftenreihe der Schweizerischen Gesellschaft für Filmwissenschaft und Filmrecht Bd. II). Schweizer Jugend Verlag, Solothurn 1963, S. 18.

4. Vgl. dazu: Kerstiens, Ludwig: Filmerziehung in den höheren Schulen. In: Zielinski, Johannes und Mitarb.: Der Spielfilm im Schulunterricht. Einführung in eine besondere Unterrichtslehre der Filmerziehung. Henn, Ratingen 1959, S. 24-30, hier S. 30.

5. Vogg, Günther: Grundlagen der Film- und Fernseherziehung. In: „Filmkunst“, Nr. 50, 1968, S. 9-13, hier S. 11.

6. Vgl. dazu: Zöchbauer, Franz: Von der Medienerziehung zur Kommunikationserziehung. In: "Jugend, Film, Fernsehen", 18. Jg., H. 1, 1974, S. 9-13, hier S. 12.

7. Vgl. dazu: Brief von Herrn Salziger vom Forschungs- und Entwicklungszentrum für objektivierte Lehr- und Lernverfahren GmbH. vom 4. Mai 1976 an den Verfasser.

8. Vgl. dazu: Lehrpläne der Bundesländer für die verschiedenen Schulstufen und -typen, soweit sie dem Verfasser zugänglich waren, aber auch: Soika, Dieter: Übersicht zum Begriff "Medienpädagogik" in den Bildungsplänen der allgemeinbildenden Schulen der BRD. In; Stuke, Franz R.; Joachim Westerbarkey (Hrsg.): Kritik der Medienpädagogik. Seminarbericht (Schriftenreihe der Landesbildstelle Westfalen H. 3). Münster 1973, S. 27-34; Treude, Burkhard: Curriculumentwicklung im Bereich Medienpädagogik, dargestellt am Beispiel allgemeinbildender Schulen in Nordrhein-Westfalen. In: Knoll, H. Joachim; Jürgen Hüther (Hrsg.): Medienpädagogik (Nymphenburger Texte zur Wissenschaft, Modelluniversität 26). Nymphenburger Verl.-Buchhandlung, München 1976, S. $52-60$.

9. Vgl. dazu: Gernert, Wolfgang: Medienerziehung in der sozialpädagogischen Ausbildung. In: "Medien- und Sexual-Pädagogik“, 4. Jg., H. 4, Oktober-Dezember 1976, S. 24-27, hier S. 25.

10. Vgl. dazu: Studienfach Medien. In: Unterrichtsmediendienst. Informationen für die Weiterbildung. 17. Lieferung, September 1977, o. S.

11. Breuer, Klaus-Detlef R.; Jürgen Hüther: Medienerziehung in der Weiterbildung. Empirische Analyse und Kritik eines Sachgebiets am Beispiel der Volkshochschularbeit. In: „Bildung und Erziehung“, 29. Jg., H. 1, Februar 1976, S. 71-80, hier S. 72.

12. Nach einem Gespräch mit Wolf Theuring anläßlich der Tagung der Deutschen Gesellschaft für Publizistik und Kommunikationswissenschaft „Medienpädagogik und Kommunikationslehre" in Freiburg i. B. am 31. 10. 1977.

13. Vgl. dazu: Kn: FK: Medienkritik im ARD-Programm. In: „Funk-Korrespondenz“, Nr. 25, 11. Juni 1976, S. 3.

14. Konzeption der medienkundlichen Sendereihe „betrifft: fernsehen“. Anlage zu Kellner, Hella: Begleitende Projektforschung zu "betrifft: fernsehen ", einer medienkundlichen Sendereihe. ZDF-Medienforschung Februar 1975, (Manus.), S. 1.

15. Lange, Willi: Zu Zielen, Möglichkeiten und Besonderheiten der Persönlichkeitsbildung durch Fernsehen. In: „Film, Fernsehen, Erziehung“. 7. Jg., H. 9 1970, S. 11-29, hier S. 16.

16. Vgl. dazu: Peschel, Edeltraud: Zur Leninistischen Konzeption von der kommunistischen Erziehung durch die Presse neuen Typs. In: „Theorie und Praxis des Sozialistischen Journalismus“, Nr. 2 1976, S. 64-71, hier S. 70.

17. Vgl. dazu: Bisky, Lothar: Massenmedien und ideologische Erziehung der Jugend. VEB Deutscher Verl. der Wissenschaften, Berlin (Ost) 1976, S. 111.

18. Bisky, Lothar; Walter, Friedrich: Massenkommunikation und Jugend. Zur Theorie und Praxis der Massenkommunikation und ihren Einflüssen auf die sozialistischen Persönlichkeitsbildung und Bewußtseinsentwicklung Jugendlicher. VEB Deutscher Verl. der Wissenschaften, Berlin (Ost) 1971, S. 111.

19. Bisky, Lothar: Massenmedien ... a.a.O., S. 113.

20. Bisky, Lothar; Süße, Heinz: Massenkommunikation und sozialistische Persönlichkeitsbildung Jugendlicher. In: „Pädagogik, Zeitschrift für Theorie und Praxis der sozialistischen Erziehung“. 28. Jg., H. 2 1973, S. 136-146, hier S. 145. 
21. Vgl. dazu: Plagemann, Karl-Ernst: Erfahrungen bei der Vermittlung filmerzieherischer Kenntnisse im Rahmen der Weiterbildung 1970/71 für Deutschlehrer. In: „Film, Fernsehen, Erziehung." 8. Jg., H. 2 1971, S. 54-67, hier S. 61.

22. Ebd., S. 57 f.

23. Peschel, Edeltraud: Zur Leninistischen Konzeption ... a.a.O., S. 64.

24. Vgl. dazu: Bisky, Lothar: Massenmedien ... a.a.O., S. 132.

25. Bundesministerium für Unterricht und Kunst (Hrsg.): Medienerziehung in den Schulen (Erlaß des BMfUuK Z. 104.785-I/10/73 vom 26. Juni 1973). In: „Verordnungsblatt für die Dienstbereiche des Bundesministeriums für Unterricht und Kunst. Wissenschaft und Forschung." Jg. 1973, 8. Stück, Nr. 94, S. 251.

26. Ebd.

27. Vgl. dazu: (Durchführungsbestimmungen) Rundschreiben Nr. 154/1973 des BMfUuK Zl. 105.645-I/10/73, Wien, 26. Juni 1973.

28. Unterrichtstechnologie. In: 589. Verordnung des Bundesministeriums für Unterricht und Kunst. Der Lehrplan der Pädagogischen Akademie und Festsetzung der neuen Unterrichtsgegenstände. Bekanntmachung des Lehrplans für den Religionsunterricht an diesen Schulen. In: „Bundesgesetzblatt für die Republik Osterreich.“ Jg. 1976, 172. Stk., 29. Oktober 1976, S. 2434, 2435.

29. In der Schriftenreihe „Veröffentlichungen des Pädagogischen und Berufspädagogischen Institutes des Bundes in Salzburg" ist im September 1977 ein Arbeitsbuch für den Lehrer erschienen: Kölblinger, Jörg; Ingrid Geretschlaeger: Massenmedien. Anregungen - Modelle - Informationen zur Medienerziehung ab der Grundschule. Ivo Haas, Salzburg 1977.

30. Vgl. dazu: Katholische Jungschar Osterreichs (Hrsg.): Erziehungs- und Bildungsziele für die außerschulische kirchliche Kinderarbeit. Wien, März 1975. Weiter: Katholische Jungschar Osterreichs (Hrsg.): Medienerziehung (JS-Studio 4). Wien 1975.

31. Vgl. dazu: fsd: „Apropos TV“ - neue medienkritische Reihe im ORF. In: „FernsehDienst", Nr. 33, 20. Juli 1976, S. 8.

32. Vgl. dazu: Institut für Unterrichtsfragen und Lehrerfortbildung (Hrsg.): Projektstudie zur Medienerziehung im Kanton Basel-Stadt, Basel 1975, (Manus.), S. 3.

33. Medienkunde. In: Interkantonaler Lehrplan für Realschulen, Nidwalden, Luzern, S. 8390, hier S. 83.

34. Vgl. dazu: Müller, Susann (AJM): Medienerziehung in den Schweizer Schulen. UNESCO, Paris, o. J. (1975), S. 1-8; Saxer, Ulridh: Konzept für eine Medienpädagogik. In: „Medienpädagogik in der Schweiz. Jahrbuch der Schweizerischen Konferenz der kantonalen Erziehungsdirektoren", Jg. 59/60, 1973/74, Huber, Frauenfeld 1975, S. 27-86.

35. Doelker, Christian: Wege zur Medienpädagogik. In: „Medienpädagogik in der Schweiz ..." a.a.O., S. 11-26, hier S. 20.

36. Ammann, Georges: "Achtung, Aufnahme“ - eine filmkundliche Sendereihe für Schüler. In: "Schweizerische Lehrerzeitung. Sondernummer: Bild und Ton im Unterricht." Nr. 25, 26. Juni 1975, S. 939-940, hier S. 939.

37. Vgl. dazu Brief von Herrn von Grüningen (Radio und Fernsehen DRS) vom 24. März 1977 an den Verfasser.

\section{S UM M A R Y}

Mass Media has become an integral part of society, so mass communication has to be taught and learnt. Education and training („Bildung ${ }^{\star}$ ) in the Federal Republic of Germany is in the hands of the Länder, wheras in Austria and in the German Democratic Republic, education is centralised, and in Switzerland, each Canton is responsible for its own system. 
In western countries there is a critical approach to the mass media, whereas in East Germany it is used for political teaching according to socialistic ideology, and the political stand regarding the media takes preference to the pedagogical approach in other countries. In none of the countries mentioned, media education is a special subject on the syllabus of compulsory education. Mass media is more or less a principle of teaching. Evidence of this is apparent in the media education projects being undertaken, except in the GDR, which are regarded as interdisciplinary, and as contributory factors in mass communication.

\section{RESUME}

Les mass media sont devenus un élément important de la société: pour cette raison, la communication de masse doit être enseignée et apprise. L'éducation et la formation sont, en République Fédérale d'Allemagne, l'affaire des "Länder"; en République Démocratique Allemande et en Austriche, les décisions sont prises de façon centralisée; en Suisse, ceci est l'affaire des cantons. Si dans les pays occidentaux on est fondamentalement critique face aux mass media, en République Démocratique Allemande on les utilise pour l'apprentissage politique de l'optique idéologique du socialisme. Là-bas, l'attitude politique des mass media jouit de la préférense sur leur attitude pédagogique. L'enseignement des mass media, en tant que matière scolaire propre dans l'école obligatoire ne s'est affirmé dans aucun pays, mais plus ou moins comme principe d'enseignement. Caractéristique pour les actuels projets pédagogiques courants (exceptée la RDA) est le fait que la pédagogie des mass media est conçue de façon interdisciplinaire et que l'aspect empiétant sur les mass media en forme de jeu de construction vers la communication des masses domine.

\section{RES UMEN}

Los medios de comunicación pasaron a ser parte esencial de la sociedad: por ello la comunicación de masas debe ser enseñada y aprendida. En la República Federal de Alemania la educación y formación son competencia de los diversos estados federados, en la República Democrática Alemana y en Austria las directrices están centralizadas, en Suiza son asunto de los Cantones. Mientras en Europa occidental se adopta una postura fundamentalmente crítica ante los medios de comunicación, en la República Democrática Alemana se emplean para la enseñanza política desde el punto de vista de la ideología socialista. Allí tienen prioridad los objetivos políticos frente a los pedagógicos. En ningún país la educación sobre medios de comunicación se abrió camino como asignatura en la escuela obligatoria, aunque sí como principio docente. Característico de los proyectos pedagógicos en curso relacionados con la comunicación es (excepto en Alemania oriental) que esta pedagogía está concebida como interdisciplinar y el aspecto específicamente comunicativo domina en forma de elemento básico para la comunicación social. 